\title{
RECOVERY OF DIFFRACTION-LIMITED OBJECT AUTOCORRELATIONS FROM ASTRONOMICAL SPECKLE INTERFEROGRAMS USING THE CLEAN ALGORITHM
}

\author{
P. W. Gorham, A. M. Ghez, C. A. Haniff, and T. A. Prince \\ Division of Physics, Mathematics, and Astronomy, California Institute of Technology, Pasadena, California 91125 \\ Received 10 April 1989; revised 26 March 1990
}

\begin{abstract}
We present a new technique for processing speckle interferometric data which uses the CLEAN algorithm, originally developed for the removal of the effects of incomplete spatial frequency coverage in aperture synthesis radio maps. Since Labeyrie first noted in 1970 that the autocorrelation of a specklegram preserves information up to the diffraction limit of an optical telescope, a number of different techniques have been applied to recover this information, usually by Fourier deconvolution of the average power spectrum of the specklegrams with a similar spectrum for a known point source. An alternative is to deconvolve the average autocorrelation of the specklegrams directly, rather than the power spectrum; this is where CLEAN, which has been shown to be a powerful tool in deconvolution problems, may be used. Also, because of the immunity of CLEAN to gaps in the spatial frequency coverage of the power spectrum, deconvolution is robust under conditions where regions of low signalto-noise ratio in the raw speckle data effectively introduce such gaps. We find that CLEAN is straightforward to apply, and yields results that exceed the quality of recoveries made using at least one other existing technique. We present diffraction-limited and near-diffraction-limited results using photonnoise-limited specklegrams taken with the Hale $5 \mathrm{~m}$ telescope at Palomar under a variety of seeing conditions.
\end{abstract}

\section{INTRODUCTION}

The principle of speckle interferometry, as applied to optical astronomy (Labeyrie 1970), is straightforward in theory. The instantaneous image $I_{\tau}(\mathbf{x})$ produced in the presence of atmospheric seeing is a convolution of the object brightness $O(\mathbf{x})$ with an instantaneous transfer function $S_{\tau}(\mathbf{x})$ :

$$
I_{\tau}(\mathbf{x})=\int O\left(\mathbf{x}^{\prime}\right) S_{\tau}\left(\mathbf{x}-\mathbf{x}^{\prime}\right) d \mathbf{x}^{\prime},
$$

where both $I_{\tau}$ and $S_{\tau}$ are stationary over an interval $\tau$ which is typically $10 \mathrm{~ms}$. The convolution becomes a product if we consider the Fourier transform of Eq. (1):

$$
\hat{I}_{\tau}(\mathbf{u})=\hat{O}(\mathbf{u}) \hat{S}_{\tau}(\mathbf{u}) \text {, }
$$

where the caret denotes the Fourier transform of the corresponding image-plane function [see Roddier (1981) for a complete discussion of $\widehat{S}_{\tau}(\mathbf{u})$ ]. If an unresolved source, represented by $O(\mathbf{x})=\delta(\mathbf{x})$, is observed under the same seeing conditions as the object of interest,

$$
I_{\tau}^{\mathrm{P}}(\mathbf{x})=\int \delta\left(\mathbf{x}^{\prime}\right) S_{\tau}\left(\mathbf{x}-\mathbf{x}^{\prime}\right) d \mathbf{x}=S_{\tau}(\mathbf{x})
$$

and

$$
\hat{I}_{\tau}^{\mathrm{P}}(\mathbf{u})=\widehat{S}_{\tau}(\mathbf{u}) \text {. }
$$

If the seeing conditions are unchanging in time over some

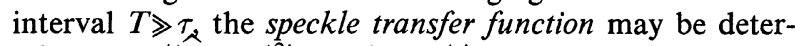
mined by $\left\langle\left|\hat{S}_{\tau}(\mathbf{u})\right|^{2}\right\rangle_{T}$, where \langle\rangle$_{T}$ denotes an ensemble average of specklegrams. The spatial power spectrum of the object is then recovered in theory by simple division (or Fourier deconvolution):

$$
|\widehat{O}(\mathbf{u})|^{2}=\frac{\left\langle\left.\widehat{I}_{\tau}(\mathbf{u})\right|^{2}\right\rangle_{T}}{\left\langle\left|\widehat{S}_{\tau}(\mathbf{u})\right|^{2}\right\rangle_{T}} .
$$

In practice, a host of problems conspire to complicate this procedure. For example, the calibration spectrum must have a much higher signal-to-noise ratio (SNR) than the object spectrum, as in any Fourier deconvolution problem, or the noise will dominate the division step in $\mathrm{Eq}$. (5). Mismatches in the seeing can introduce systematic mismatches in the calibration and object spectrum, leading to nonlinear effects that are difficult to correct once the division has been performed. Even when the effects of changing seeing are ignored, the calibration spectrum is never noise free; for example, in photon-counting detectors, the effects of quantization noise can produce zeros in the power spectrum of the calibrator, thus giving nonphysical amplitudes for the calibrated object spectrum. Often a filtered spectrum of the calibrator is used, but criteria for the type of filter or the cutoff frequency are difficult to determine objectively.

Recently, progress has been made in improving the reliability of Fourier deconvolution for speckle interferometry under conditions where simple division of spectra becomes impractical; for example, due to the low SNR of the data. Ebstein (1987) has developed an iterative Fourier deconvolution technique which uses a series of projections onto convex sets to successively constrain an initial estimate of the object spectrum, with requirements such as positivity and symmetry in the object power spectrum and positivity of the corresponding autocorrelation function (ACF).

Here we present an alternative technique which employs CLEAN algorithm to perform the deconvolution entirely in the domain of the specklegram average ACF. If the raw data are reasonably free of artifacts, the technique may be applied to recover a diffraction-limited autocorrelation of the observed astronomical object, even if the SNR of the data is of order unity or less throughout large portions of the raw object power spectrum obtained through the averaging of the spectra of the individual specklegrams. Fourier amplitudes are then obtained by the Fourier transform of the recovered ACF.

The primary differences between CLEAN and techniques such as the convex projection (CP) algorithm introduced above, which iterate between the power spectral and ACF domains, arise in the way the data are constrained during the 
application sequence. We will discuss these differences in more detail in a later section. In the following section we introduce a formalism for image-autocorrelation deconvolution, and describe the CLEAN algorithm as it applies to this problem. In later sections we present results using CLEAN, and a comparison of the performance of CLEAN with that of the $\mathrm{CP}$ algorithm, using speckle data obtained with the Hale $5 \mathrm{~m}$ telescope.

\section{APPLICATION OF CLEAN TO SPECKLE INTERFEROMETRY} DATA

The success of the CLEAN algorithm (Hogbom 1974) in improving the quality of maps made of aperture synthesis in radio astronomy is well established. Its behavior is generally predictable, both when it succeeds and when it fails. Reviews of the algorithm and improvements to it may be found in Cornwell (1983, 1986), Clark (1980), and Steer, Dewdney, and Ito (1984).

CLEAN works in an intuitively simple way: assuming the object is represented as a sum of point sources of varying intensity and position, each convolved separately with the "dirty beam" convolution function $D(\mathbf{x})$, it subtracts a fraction $\gamma$ of the dirty beam from the maximum point in the image (or "dirty map"), recording separately the position $\mathbf{x}_{i}$ of the maximum, and $\gamma D\left(\mathbf{x}_{i}\right)$, the amount subtracted. The process is repeated until the maxima fall into the noise or begin to appear outside the known region of emission. The list of maxima and their accumulated intensities are then convolved again with a resorting "clean" beam which is usually chosen to be the central lobe of the dirty beam, and the residuals are added back into the map. The factor $\gamma$, called the loop gain, may be adjusted depending on the type of object; an extended, low-contrast object will generally require a smaller $\gamma$ than an object consisting of a number of bright pointlike features.

To see how CLEAN applies to speckle data, the sky brightness distribution of the object in the telescope image plane is represented by a delta-function decomposition, convolved with the telescope beam. The delta functions can be taken as single pixel intensities in an imaging device, if the object were viewed with an ideal telescope and detector with no noise. For simplicity in what follows, we take the telescope beam to be a stationary part of the transfer function; including it explicitly does not change the analysis. Thus we write

$$
O(\mathbf{x})=\sum_{p=1}^{M} J_{p} \delta\left(\mathbf{x}-\mathbf{x}_{p}\right)
$$

with $2 M$ unknowns $J_{p}$ and $\mathbf{x}_{p}$. The convolution of Eq. (1) then becomes

$$
I_{\tau}(\mathbf{x})=\sum_{p=1}^{M} J_{p} S_{\tau}\left(\mathbf{x}-\mathbf{x}_{p}\right) .
$$

As Labeyrie (1970) originally showed, the ACF of the image, rather than the image itself, is the good observable that may be averaged. A more complete discussion of this may be found in Greenaway and Dainty (1980).

The ACF of the image is

$$
\begin{aligned}
A_{\tau}^{I}(\chi) & =\int d \mathbf{x} I_{\tau}(\mathbf{x}) I_{\tau}(\mathbf{x}+\chi) \\
& =\int d \mathbf{x}\left(\sum_{p=1}^{M} J_{p} S_{\tau}\left(\mathbf{x}-\mathbf{x}_{p}\right)\right)
\end{aligned}
$$

$$
\begin{aligned}
& \times\left(\sum_{q=1}^{M} J_{q} S_{\tau}\left(\mathbf{x}-\mathbf{x}_{q}+\chi\right)\right) \\
= & \sum_{p=1}^{M} \sum_{q=1}^{M} J_{p} J_{q} \\
& \times \int d \mathbf{x} S_{\tau}\left(\mathbf{x}-\mathbf{x}_{p}\right) S_{\tau}\left(\mathbf{x}-\mathbf{x}_{q}+\chi\right),
\end{aligned}
$$

where the integral extends over the entire image for each frame. With a change of variable, $\mathbf{x} \rightarrow \mathbf{x}+\mathbf{x}_{p}$, the integral within the double summation may be seen to be the ACF of the instantaneous transfer function itself. Averaging over all frames gives

$$
\left\langle A^{I}(\chi)\right\rangle_{T}=\sum_{p=1}^{M} \sum_{q=1}^{M} J_{p} J_{q}\left\langle A^{S}\left(\mathbf{x}_{p}-\mathbf{x}_{q}+\chi\right)\right\rangle_{T} .
$$

If the object is a single point source at the origin with intensity $J_{0}$,

$$
O(\mathbf{x})=J_{0} \delta(\mathbf{x}),
$$

then the average ACF for the corresponding set of pointsource speckle frames is

$$
\left\langle A^{P}(\chi)\right\rangle_{T}=J_{0}^{2}\left\langle A^{S}(\chi)\right\rangle_{T} \text {. }
$$

The ensemble average ACF of the right-hand side of Eq. (13) may be seen as the ACF-domain analog to the speckle transfer function mentioned in Sec. I.

Consider now the ACF of the object distribution in Eq. (6):

$$
A^{o}(\chi)=\sum_{p=1}^{M} \sum_{q=1}^{M} J_{p} J_{q} \delta\left(\mathbf{x}_{p}-\mathbf{x}_{q}+\chi\right)
$$

Comparing Eqs. (11) and (14), it is evident that the ensemble average ACF of the image if the object ACF (still a set of discrete delta functions), convolved with the speckle transfer ACF. This is to be expected by the Fourier transform relationship between $\mathrm{ACF}$ and power spectrum, and the result of Eq. (3). Now, let $J_{p} J_{q}=\alpha_{p q} J_{0}^{2}$ for some set of coefficients $\alpha_{p q}$. Using Eqs. (11) and (13), we obtain

$$
\left\langle A^{I}(\chi)\right\rangle_{T}=\sum_{p=1}^{M} \sum_{q=1}^{M} \alpha_{p q}\left\langle A^{P}\left(\mathbf{x}_{p}-\mathbf{x}_{q}+\chi\right)\right\rangle_{T},
$$

which states that any image ACF may be expressed as a sum of point source ACFs with appropriate scaling coefficients $\alpha_{p q}$ and offsets $\mathbf{x}_{p}-\mathbf{x}_{q}$. Equation (15) forms the basis for the use of CLEAN in astronomical speckle interferometry. The algorithm makes estimates of the positions $\mathbf{x}_{p}-\mathbf{x}_{q}$ and the coefficients $\alpha_{p q}$ for the object ACF by the iteration described at the beginning of this section.

A standard set of criteria for convergence of CLEAN has been given by Schwarz $(1978,1979)$. Applied to the ACF data discussed here, these conditions are:

(a) The beam must be symmetric; thus

$$
\left\langle A^{P}(\chi)\right\rangle_{k}=\left\langle A^{P}(-\chi)\right\rangle_{k} \text {. }
$$

(b) The beam must be positive definite or positive semidefinite:

$$
\left\langle A^{P}(\chi)\right\rangle_{k} \geqslant 0 \text {. }
$$

(c) The dirty ACF image must not contain significant power at spatial frequencies above the highest spatial frequency present in the beam: 


$$
\frac{\left\langle\left|\widehat{I}_{\tau}(\mathbf{u})\right|^{2}\right\rangle_{T}}{\left\langle\left|\widehat{I}_{\tau}(0)\right|^{2}\right\rangle_{T}} \leqslant \frac{\left\langle\left.\widehat{I}_{\tau}^{P}(\mathbf{u})\right|^{2}\right\rangle_{T}}{\left\langle\left|\widehat{I}_{\tau}^{P}(0)\right|^{2}\right\rangle_{T}} \text { for all } \mathbf{u} .
$$

It is clear that Eq. (16) is satisfied by definition for the ACF of any function. Equation (17) is also satisfied for any ACF determined by image-plane correlation (rather than by a Fourier transform of a power spectrum), since it must be positive or zero. Equation (18) will be satisfied if the seeing does not significantly change between the observation of the point source and the object; any resolved source must satisfy Eq. (18) again be definition. In photon-noise-limited data, of course, there is quantization noise due to the discrete nature of the detection process. The effect of this is to produce a residual noise throughout both the beam and object power spectra (corresponding to a residual shot-noise level in the ACF) which effectively defines the background noise level for any speckle technique. Equation (18) is weakly violated by this effect, but the residual power is not "significant" because it is not a threat to the convergence of CLEAN except in the obvious case where it exceeds the level of the signal itself throughout the spectrum. CLEAN will of course fail in such cases simply because there is very little signal to recover.

We regard these conditions as satisfied in principle for speckle interferometry data. However, we note that detector systematics which do not involve a convolution process, such as image-plane scale nonlinearities, or fields that are not flat, cannot be removed by CLEAN. We discuss briefly the systematics specific to our own detector in a later section.

\section{DATA REDUCTION ALGORITHM}

This section describes the practical details of application of CLEAN to actual data. Although the basic principles of the techniques described are general, the discussion is limited to data for which the image-plane position and arrival time of discrete photon events have been recorded.

The initial step is to calculate an unbiased estimator of the ACF. For data consisting of discrete photon events, where

$$
I_{\tau}(\mathbf{x})=\sum_{p=1}^{M} \delta\left(\mathbf{x}-\mathbf{x}_{p}\right),
$$

accumulation of the position difference vector for all parts of different events (including events which arrive at the same position at different times) within a single frame yields an unbiased ACF which is positive semidefinite. The photonnoise bias (see Dainty and Greenaway 1979) is avoided by not correlating each photon with itself. Thus

$$
A_{\tau}^{I}(\chi)=\sum_{p=1}^{M} \sum_{q=1}^{M} \delta\left(\mathbf{x}_{p}-\mathbf{x}_{q}+\chi\right), \quad p \neq q .
$$

The ACF for all frames is then accumulated and averaged. A $50 \%$ overlap in the frame boundaries will improve the SNR, and even further improvement may be achieved by using a running frame, or ring buffer, where successive events are correlated with all events that precede them within the chosen frame interval. Observations of a point source calibration star, or a number of them, must be done as often as possible. Assuming that inspection of the separate calibrator ACFs indicates them to be consistent in seeing with the object ACF, they may be summed to improve the SNR.

If the accumulated calibrator ACF has significantly more (or less) total power than the object ACF, it should be scaled to make them roughly equal. Exactly equal power is not necessary, since the loop gain of CLEAN will take care of small scaling differences for the most part. We have experimented with various loop gains, and we find that for simple discrete objects such as binary or multiple stars, a loop gain of $\sim 0.1$ is reasonable. For more extended objects of lower contrast, lower loop gains are more effective, since the extended structure tends to be thinly spread over the shoulders of the seeing disk ACF, and larger loop gains tend to miss this structure. Experimentation is necessary, and the literature gives little strong guidance on this issue, other than a general feeling that loop gains $\ll 1 \%$ rarely give much improvement in the results. However, it appears to be necessary in general to use lower loop gains than in the corresponding case in radio astronomy, because the desired structure in the ACF is generally a small fraction of the seeing-dominated intensity.

Since CLEAN does not inherently guarantee symmetry in the recovered ACF, this may be enforced during the cleaning by requiring that each component found and subtracted be followed by an equivalent point-symmetric component subtraction. An alternative is to use CLEAN as is, and then symmetrize the final ACF by averaging the point-symmetric components. The two methods appear to produce virtually identical results. Even without requiring symmetry at all, we find that it is never violated by more than $\sim 1 \%$ in the final ACF. The cleaning process is terminated when the maximum found either (a) falls below a chosen threshold, usually determined by the measured statistical noise in the ACF, or (b) falls outside the known region of emission, again indicating that the noise in the ACF is now comparable to any remaining signal. Cleaning far into the noise does not usually yield any improvement to the final map, and can actually degrade the recovery by introducing a "bowl" in the final ACF.

The final stage of the process is the convolution of the derived CLEAN components with the restoring beam, and the addition of the residuals to the map. We examine two criteria in determining the shape of the restoring beam: (a) the raw power spectrum $\left\langle\left|\widehat{I}_{\tau}^{P}(\mathbf{u})\right|^{2}\right\rangle_{T}$ of the calibration star, and (b) the raw power spectrum $\left\langle\left|\hat{I}_{\tau}(\mathbf{u})\right|^{2}\right\rangle_{T}$ of the object being cleaned. A comparison of two spectra is necessary to confirm that Eq. (18) is satisfied. Beyond this, if the object is a multiple star, the spatial frequency radius beyond which the spectrum shows no significant power is used to define the width of a corresponding Gaussian restoring beam, which is $\sqrt{2}$ wider (FWHM) than the image beam, since the restoration involves the ACF of the image. For cases where the spectrum indicates it is necessary, an elliptical Gaussian may be employed to match the calibrator spectrum, since the visibility modulation in the object spectrum may distort the contours at the highest spatial frequencies. However, for multiple star systems the restoring beam is not an important factor in the resulting cleaned $\mathrm{ACF}$, since the parameters of interest do not depend on the exact beam shape, unless the stars are barely resolved.

For extended objects, however, the restoring beam shape is important, and more effort should be made to ensure that it corresponds to the data as well as possible. In this case, the object spectrum must be used to determine the cutoff frequency. The restoring beam should have a width which is consistent with the highest possible spatial frequency present in the object spectrum. Once this frequency has been approximately established, the calibrator spectrum may be used to determine parameters of an elliptical Gaussian 
which is consistent with the telescope response function at this frequency.

\section{RESULTS}

The photon-counting camera and its associated electronics, and the observations reported here, are described in detail in Gorham et al. (1989) and Nakajima et al. (1989). The initial data reduction (calculating the ACF of raw data) was done on a 512-processor NCUBE concurrent supercomputer at Caltech, which uses the hypercube interconnection topology (see, for example, Fox et al. 1988). A typical dataset such as those for which we present results here contained $\sim 6 \times 10^{6}$ photon events, recorded at rates of between $1.5 \times 10^{4}$ and $5 \times 10^{4}$ events per second. The duration of the frames was between 3 and $30 \mathrm{~ms}$, depending on seeing, thus giving $\sim 50-1500$ photon events per frame. A typical reduction for both calibrator and object required $\sim 1 \mathrm{hr}$ on the NCUBE or $\sim 16 \mathrm{hr}$ on a SUN 4/360 for comparison. One advantage of using the NCUBE was that it allowed iteration of the ACF computation for a number of different integration times, to optimize this important parameter.

In the application of CLEAN to these data the raw object ACF was vignetted to the central $128^{2}$ pixels $( \pm 0.48$ arc$\mathrm{sec}$ ), and the calibrator ACF to $256^{2}$ pixels since it must be nominally twice the size of the map being cleaned. Standard Hogbom CLEAN was used, with a single window. In some cases a $256^{2}$ map and a $512^{2}$ beam were used for consistency checks. Once the raw ACFs are prepared, any standard CLEAN code could be used, for example those available in the VLBI and AIPS software packages. We have not yet evaluated any of the features of improved CLEAN algorithms, such as the Clark algorithm (Clark 1980).

This report presents only data relating to the amplitudes or ACFs of the objects observed; in an additional paper (Gorham et al. 1989) imaging results are reported which make use of the recovered ACF data discussed here, and phases reconstructed through bispectral analysis.

We note that all of our observations were affected to some degree by detector bias. The photon-counting camera em-

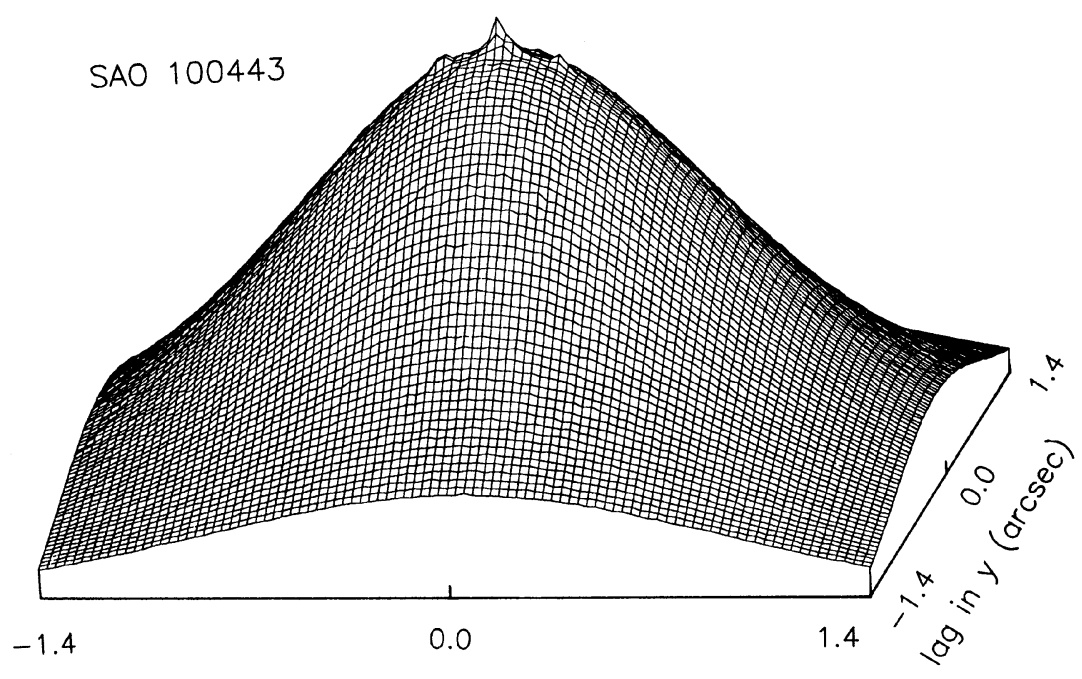

(a)

$\operatorname{lag}$ in $x(\operatorname{arcsec})$

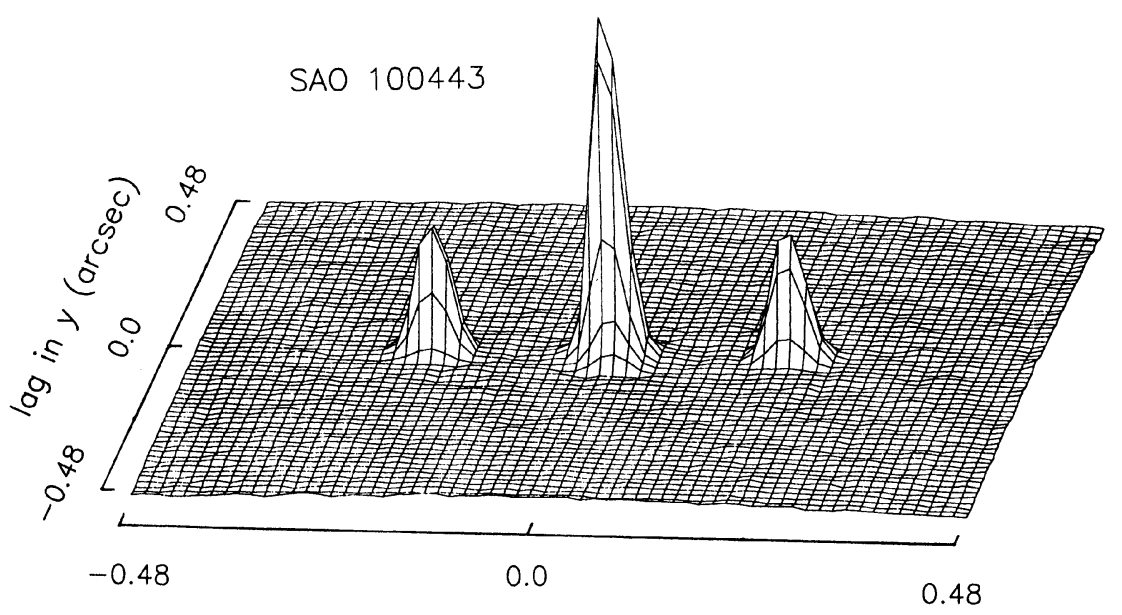

(b)

$\operatorname{lag}$ in $x(\operatorname{arcsec})$
FIG. 1. (a) Raw autocorrelation function (ACF) of SAO 100443 with seeing of $\sim 1.7$ arcsec during the observation. The ACF appears narrower than this because image wander is effectively removed. The data have been smoothed slightly in the process of convolving onto this grid. The vertical scale and orientation are arbitrary. (b) Estimate of the true object ACF constructed by CLEAN, with $\sim 20$ iterations. Orientation is the same as in (a), but vertical and horizontal scales are adjusted to show detail. 
ployed a Ranicon, which uses a high-gain microchannel plate electron multiplier read out by a resistive anode charge divider [see Clampin et al. (1988), and references therein for a description of these detectors]. This type of detector I suffers from a sensitivity bias where by a pixel remains dead for some time after recording an event, with time constants of tens of milliseconds possible in some cases. The effect is complicated by a tendency for the electric field between the stages of the microchannel plate to realign itself, resulting not simply in a loss of light due to saturation, but in a refocusing of the electron multiplier, and a corresponding nonlinear distortion of the image at spatial wavenumbers of $\sim 0.1$ pixel $^{-1}$ or more. This problem, and a partial correction which can be made to compensate for it in the raw data, is discussed in more detail in Gorham et al. (1989). The result of this effect was that the errors on estimates of the magnitude differences for the binary star systems were increased somewhat, since the level of saturation will be different for the two components of a binary star system. The effect also produces a loss of power at high spatial frequencies for extended objects, because the local distortion causes overlap in adjacent, low-contrast image components. This detector bias, though unfortunate, does not change our assessment of the effectiveness of CLEAN in recovering the ob- ject ACF in the absence of such detector-specific problems.

The binary star observation were made in April 1988. Figures 1-4 show, respectively (a) the object raw ACF including the seeing effects and (b) the recovered ACF, for four binary stars. The scale changes between (a) and (b) in each case, to show the larger-scale structure of the seeing-convolved ACF; however, the orientation of each plot is consistent from (a) to (b). The seeing varied from 1.7 arcsec for SAO 100443 (Fig. 1) to 1.1 arcsec for SAO 45685 (Fig. 4), and in a number of cases the scale of plot (a) varies from star to star because of the seeing differences. SAO 82390 (Fig. 3) is listed as a speckle interferometry calibration standard by McAlister and Hartkopf (1984); the recovered ACF of Fig. 3 (b) indicates a magnitude difference of $\Delta m=2.3 \pm 0.1$ mag, consistent with McAlister's value. The observation times for all stars ranged from 2 to $5 \mathrm{~min}$. Contemporaneous measurements of all of the stars presented here may be found in McAlister and Hartkopf (1988).

To partially quantify the character of the recovered ACFs, we use the dynamic range, defined as the ratio of the recovered intensity of the primary component to that of the largest noise peak. This quantity, which gives an indication of the value of the recovered ACF in determining characteristics of components that are faint relative to the brightest
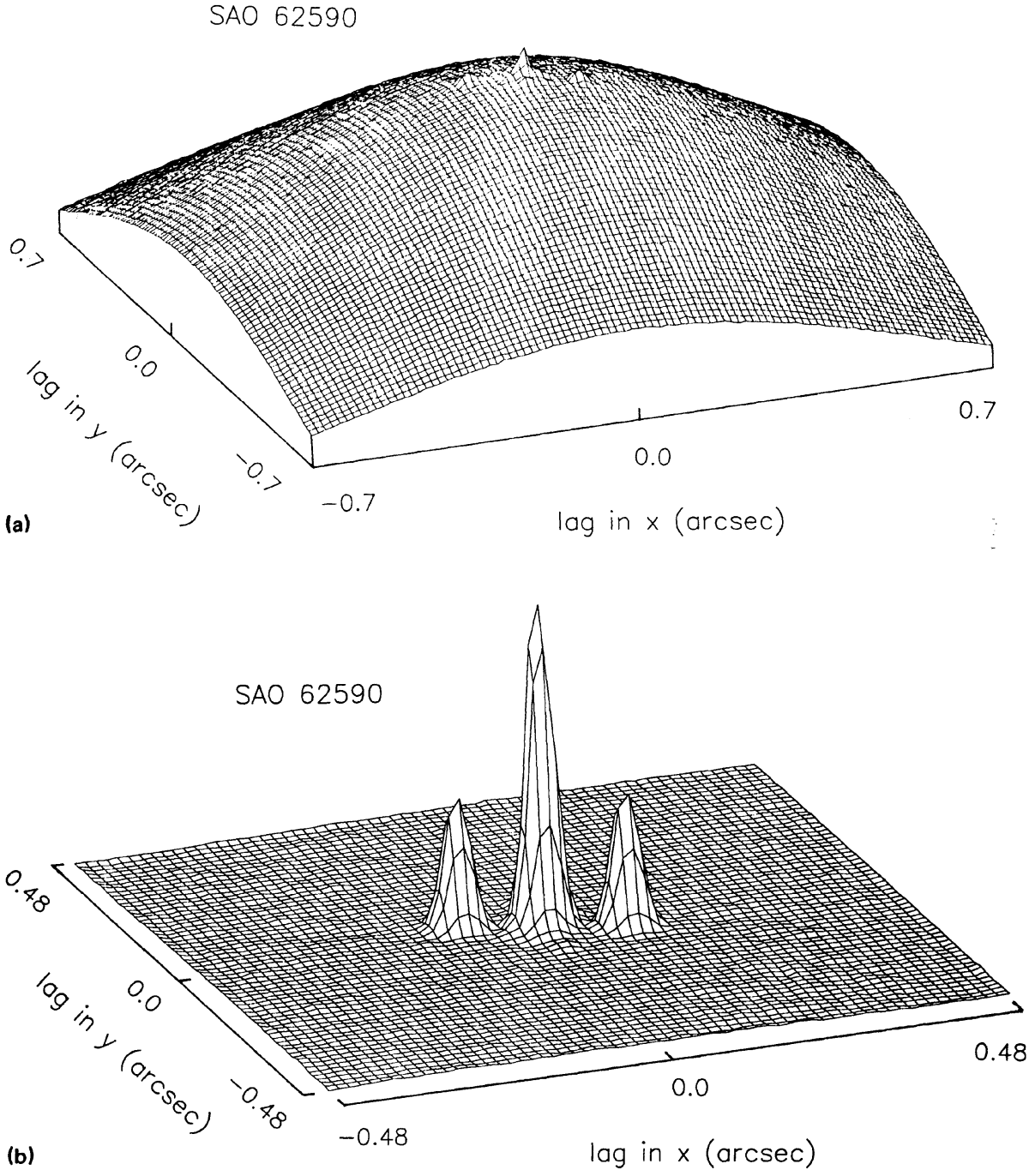

Fig. 2. (a) Similar to Fig. 1, for SAO 62590 , with seeing of $\sim 1.3$ arcsec, and horizontal scale enlarged to show detail. (b) Recovered ACF, showing a 0.11 arcsec binary system with $\Delta m=0.94$ mag. 

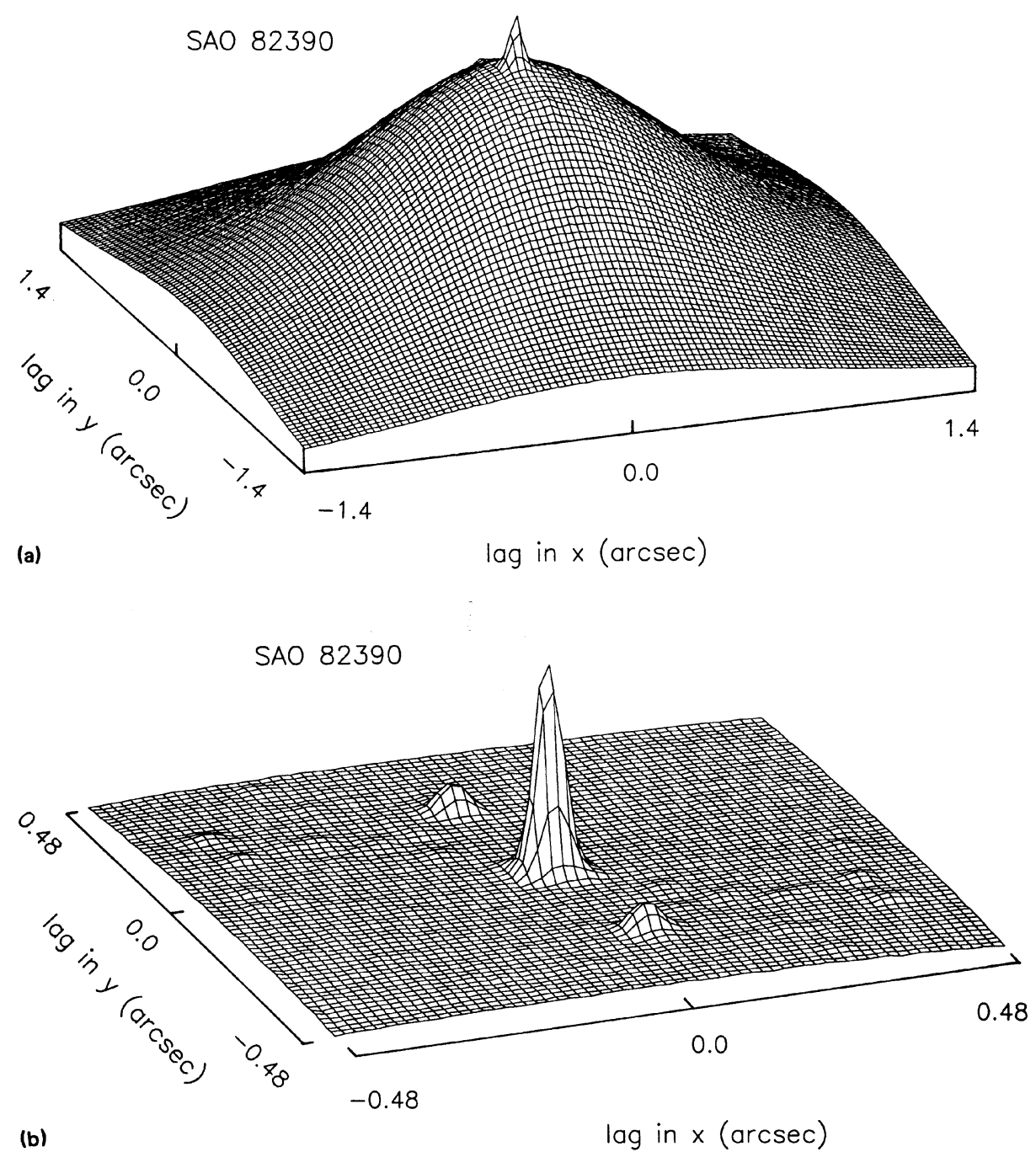

FIG. 3. (a) Similar to Fig. 1, for SAO 82390 , with seeing of $\sim 1.2$ arcsec. (b) Recovered ACF for this star confirms the magnitude difference $\Delta m=2.4$ mag reported by McAlister and Hartkopf (1984). point, must depend both on the SNR of the raw data and on the characteristics of the algorithm used to deconvolve the seeing effects. Dynamic range also has a dependence on the spatial frequencies associated with the recovered component in question. We find that, at the spatial frequencies corresponding to the diffraction limit of the $5 \mathrm{~m}$ telescope ( 30 $\operatorname{arcsec}^{-1}$ at $630 \mathrm{~nm}$ ), a dynamic range of as much as $\sim 70: 1$ is achieved in these cleaned ACFs, indicating that the technique is capable of detecting contrasts of $\geqslant 4.5 \mathrm{mag}$ under the best conditions. The average high-frequency dynamic range for all of the binary star recoveries was $\sim 40: 1$. At low spatial frequencies, CLEAN would occasionally leave residual bowls or saddles. However, in most cases, the recovered background level gave a low-frequency dynamic range equal to or higher than that at the diffraction limit.

We note that Airy rings (or secondary diffraction maxima), which one would expect to see at these dynamic range levels, are naturally suppressed in the cleaning process, since CLEAN treats these as part of the "dirty map," and removes them as it would any other artifact. We have recovered such secondary maxima in similar data, with patterns and amplitudes expected for the aperture used, by cleaning with beams that were modified to retain this structure.
In Fig. 5 we show the results of CLEAN on the extended object, minor planet 2 Pallas, which subtended $\sim 0.25$ arcsec during this observation, in July 1988, when the asteroid was at a distance of $\sim 2.5 \mathrm{AU}$, at $m_{R}=9.4 \mathrm{mag}$. The apparent size of the seeing disk during this observation was $\sim 1$ arcsec; however, the integration time was very short compared to those of the April observations, with frame integration times of $\leqslant 3 \mathrm{~ms}$ determined by checking visibilities for a number of integration times. The event rate was thus $\sim 60$ events per frame, and the observation lasted for about $15 \mathrm{~min}$.

Figure 5(a) shows the raw ACF, smoothed slightly to suppress the statistical photon noise. The recovered ACF in Fig. $5(\mathrm{~b})$ required $\sim 100$ loop iterations of CLEAN. The CLEAN loop gain used here was lower $(\sim 2 \%)$ than for the binary stars. The restoring beam corresponded to an image resolution of $\sim 0.1$ arcsec, rather than the diffraction-limited value of $\sim 0.03$ arcsec, since the power spectrum did not indicate any higher-resolution information. This loss of the high spatial frequencies appeared to be due in part to the saturation problem discussed above, and the problem was evident in the data at scales of $\sim 60$ milliarcsec and below, where the distortion peculiar to the microchannel plate detector could be seen. The effect did not degrade the data at 


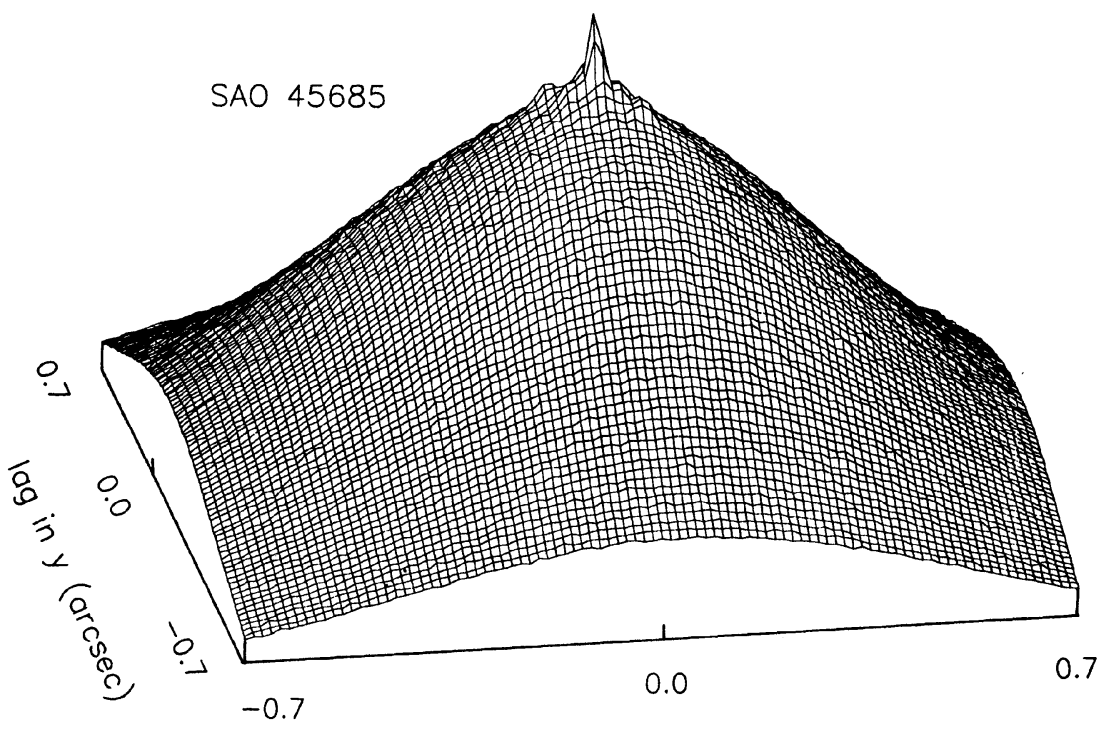

(a)

$\operatorname{lag}$ in $x(\operatorname{arcsec})$
FIG. 4. (a) Similar to Fig. 1, for SAO 45685 , with seeing of $\sim 1.1$ arcsec. (b) Recovered ACF, showing this 0.084 arcsec binary system with $\Delta m=2.2 \mathrm{mag}$.

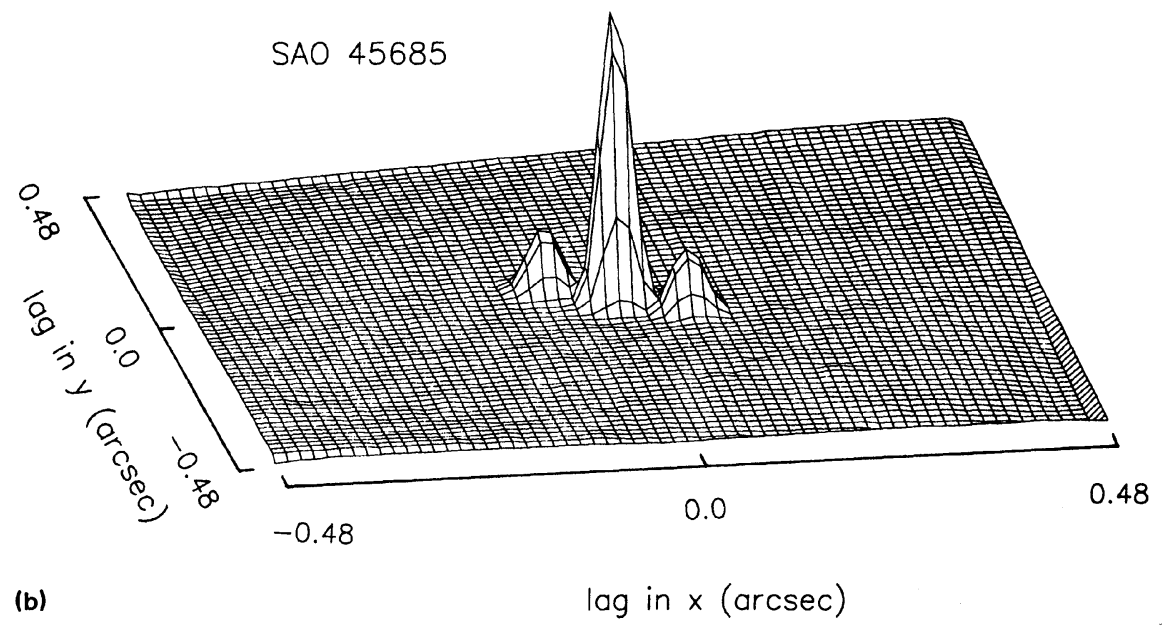

scales larger than this. The recovery was imperfect at the lower spatial frequencies, which may be seen in the low-level mound upon which the asteroid ACF appears to sit. At the intermediate spatial frequencies, the dynamic range is $\sim 15: 1$.

The average projected diameter of the asteroid derived from this ACF is $530 \pm(50,15) \mathrm{km}$, where the standard statistical and systematic errors, respectively, are in parentheses. This value is consistent with those derived from radiometric methods (Lebofsky et al. 1986), IRAS (Matson et al. 1986), and other speckle interferometry results (Drummond and Hege 1988), all of which give a mean diameter in the range $523-532 \mathrm{~km}$.

\section{DISCUSSION AND COMPARISON TO CP ALGORITHM}

As mentioned in the Introduction, the primary differences that arise between CLEAN and iteration techniques such as

the CP method come from the way the constraints enter the processing sequence. Naively, one might expect that the CLEAN result should be compared to the initial estimate (using division of the object spectrum by a calibrator spectrum) in the $\mathrm{CP}$ algorithm, since both represent the result of what is nominally a pure deconvolution process. However, this is not correct since CLEAN in fact also implicitly interpolates in the spatial frequency domain over those regions for which there is inadequate u-plane coverage (Cornwell 1986).

This implicit interpolation is of course why CLEAN is so successful in improving radio synthesis maps, in which the uplane coverage of the dirty map is often very sparse. In fact, the same type of interpolation is effective in speckle data, where the sparsity of u-plane coverage is caused not by the shape of the aperture (as in the radio case) but rather by the loss of visibilities due to inadequate SNR, or even systematics which corrupt portions of the otherwise filled $\mathbf{u}$ plane. It is CLEAN's immunity to gaps in the visibility function, as well 

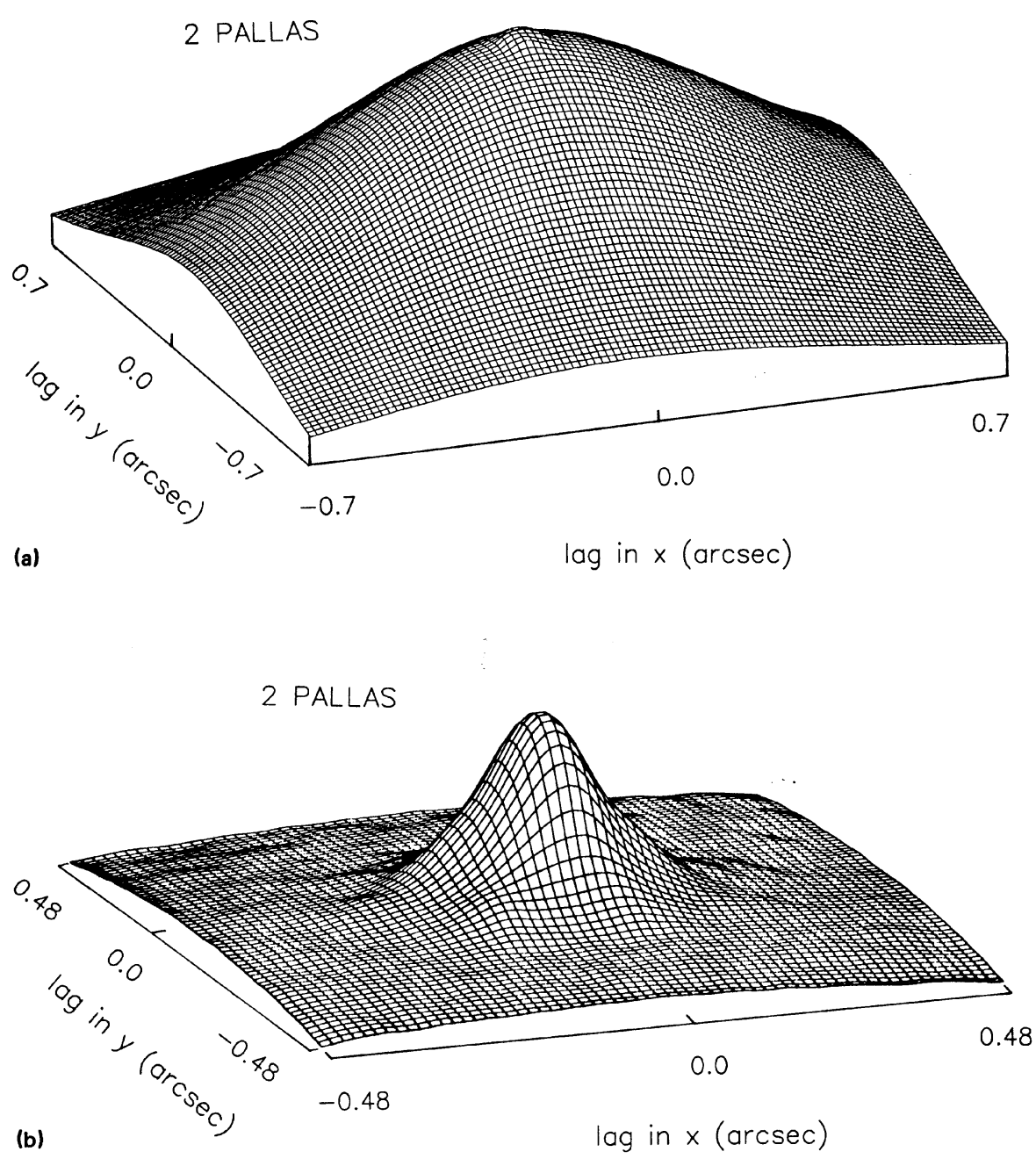

FIG. 5. (a) Raw intensity ACF (smoothed slightly) of asteroid 2 Pallas, with $\sim 1$ arcsec seeing but very short $(\sim 3 \mathrm{~ms})$ atmospheric coherence times. (b) Recovered intensity ACF of Pallas, restored with an $\sim 0.1$-arcsec-equivalent beam, as described in the text. The average diameter derived from these data is $\sim 530 \mathrm{~km}$. as its power as a deconvolution technique, which makes it a natural choice to process noisy speckle data.

In the CP algorithm, the gaps in the visibility function of the initial spectral estimate are gradually filled in by a sequence of constraints that are applied in both the power spectral and ACF domains, such as (a) positivity of the spectrum, (b) a width and shape of the point-spread function which is commensurate with the data quality and telescope used, (c) a spectrum which is statistically compatible with the initial estimate, (d) positive $\mathrm{ACF}$, and (e) nonzero ACF over only a finite support region, usually determined by some prior knowledge of the source. The iteration then proceeds by Fourier transforming back and forth between the ACF and spectral domains, applying the appropriate constraints at each iteration, until convergence is observed.

In our application of CLEAN, only (b) and (c) above are explicitly addressed, through the choice of the restoring beam. Typically, the choice of restoring beam also affects (a), since a badly corrupted central lobe may introduce some negative power. Also, if the spectrum of the residuals contains negative power, it may appear in the final cleaned $\mathrm{ACF}$ as well. The stopping criterion will usually guarantee (d), but seeing mismatches between calibrator and object can lead to problems here, usually in the form of low-level bowls or saddles across the resulting cleaned ACF, a problem which is also seen in radio applications.

In Fig. 6(a) we show a raw power spectrum of the data used for recovery of the ACF of SAO 100443 as shown in Fig. 1(b). The first eight contours are given in intervals of twice the root-mean-square deviation from the mean, as measured in the outer edges of the spectrum, and the remaining contours are logarithmic from $0.1 \%$ of the maximum to the maximum. The theoretical cutoff frequency of the telescope occurs at a radius of $\sim 30$ cycles/arcsec from the origin. In Figs. 6(b) and 6(c), we show the results of (b) ACF recovery using the convex projection algorithm of Ebstein (1987) and (c) the results of our application of CLEAN, both as contour plots, with intervals at $\pm 1, \pm 2,+4,8,16,32$, 64 , and $99 \%$ of the maximum. Negative contours (which are not allowed by the $\mathrm{CP}$ algorithm) are shown as dashed lines. Ignoring the artifact adjacent in $x$ to the central component in Fig. 6(b), which is almost certainly due to the saturation effects of our detector, the dynamic range of Fig. 6(b) is $\sim 40: 1$ and that of Fig. 6 (c) $\sim 70: 1$. 
A more significant difference between the two recoveries is the difference in the estimate of $\Delta m_{R}$ given by the ACFs. CLEAN gives $\Delta m_{R}=1.0 \mathrm{mag}$, while $\mathrm{CP}$ gives $\Delta m_{R}=1.6 \mathrm{mag}$. As discussed in more detail in Gorham et al. (1989), this system is cataloged as an F5 V + F5 V pair. The spectra are blended, however, and some magnitude difference between the components is possible. A difference of five spectral subclasses between the components is necessary to achieve $\Delta m_{R}=1.0 \mathrm{mag}$. However, a difference of $\Delta m_{R}=1.6 \mathrm{mag}$ implies an entire spectral class difference between the two components, which appears unlikely for this system. Nisenson (1988) has also noted the tendency for the CP algorithm to have problems with the accuracy of its amplitude recovery.

In one other case we had data on a star for which there was independent spectral information: $\beta$ LMi (HR 4100). The results of the analysis of these data are shown in Fig. 7, in the same sequence and with the same contour levels as Fig. 6. The SNR in the raw data was even lower than in SAO 100443 , and the recoveries of the two techniques both yielded ACFs of dynamic range significantly lower than in other cases. In this case, however, the dominant noise in the $\mathrm{CP}$ recovery is an artifact around the central lobe, which does not appear in any form in the CLEAN recovery. The CP recovery again gave a $\Delta m$ which was larger than that of the CLEAN result by about $0.1 \mathrm{mag}$, but the spectra information here cannot constrain the $R$ magnitude difference well enough to decide which, if either, is correct, and the statistics are obviously poorer than in the case of SAO 100443.

Another notable difference between the CP and CLEAN recoveries is the tendency for the noise to be concentrated toward the maximum of the image in the $\mathrm{CP}$ recovery; this may be attributed to constraint (e) above, which zeros all the intensity outside a user-specified support region. In this case we specified a circle of radius equal to half the image size as the support; the corner regions were thus effectively zeroed at each iteration. Softening this constraint to allow emission in the corners as well would be equivalent to no constraint at all. Thus the uneven noise distribution appears to be the price that is paid for using this ACF support constraint. ACF support constraints may also be used in a slightly weaker way in CLEAN by terminating the process when the maxima found fall outside of the support; we have not used such a constraint in this analysis.

In all other binary star data which we reduced with the two algorithms, the magnitude difference recovered by CP systematically exceeded that recovered by CLEAN. We attempted to track the source of this discrepancy by following the magnitude ratio of SAO 100443 at each step of the CP iteration. It was found that, although the initial few iterations gave a $\Delta m$ consistent with the CLEAN result, the remaining iterations continued to remove power from the secondary component until the result converged, after about ten iterations. The reason for this appears to be due to the fact that the algorithm does not attempt to conserve the total power in either the ACF or power spectral domains. This is most evident in the constraint of ACF support, which discards all flux outside a certain region in the ACF image. In addition, the thresholding of components at zero in both the $\mathrm{ACF}$ and spectral domains, and the statistical clipping in the

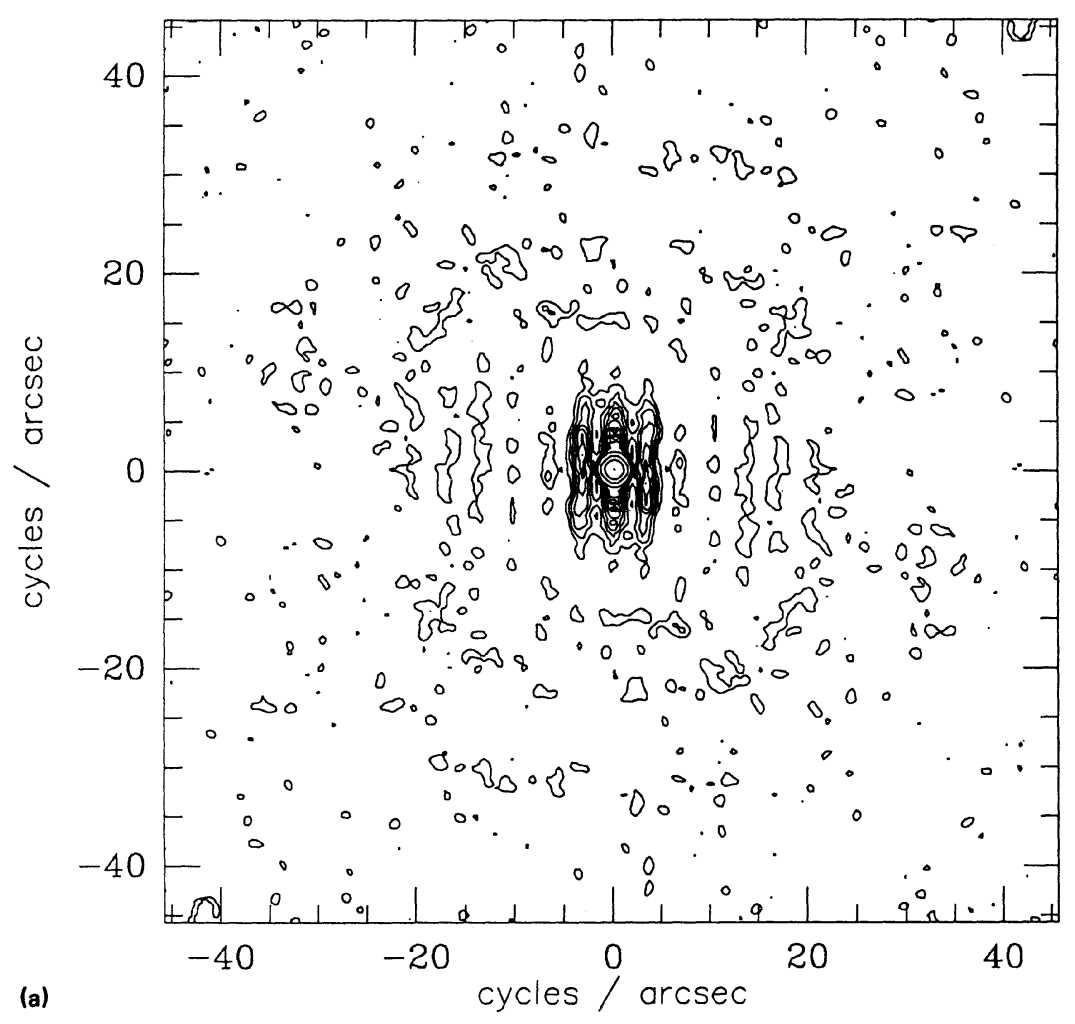

Fig. 6. (a) Ensemble average power spectrum of the raw specklegrams of SAO 100443 (same star as in Fig. 1). The first eight contours begin at twice the rms noise in the spectrum and continue at that interval, then in logarithmic intervals of $0.001,0.01,0.1$, and 0.99 times the maximum value. (b) Estimated ACF of the star, recovered using the convex projection algorithm (Ebstein 1987). Contours are at $1 \%, 2 \%$, $4 \%, 8 \%, 16 \%, 32 \%, 64 \%$, and $99 \%$ of the maximum. The dynamic range here is about 40:1, ignoring the artifact near the central lobe, which is almost certainly due to detector systematics. (c) CLEAN estimate of the ACF, with contours at $\pm 1 \%, \pm 2 \%, 4 \%, 8 \%, 16 \%$, $32 \%, 64 \%$, and $99 \%$ of the maximum, and a dynamic range of 70:1. The CLEAN result also shows some distortion of the central lobe. 
303 GORHAM ET $A L$.: SPECKLE INTERFEROGRAMS

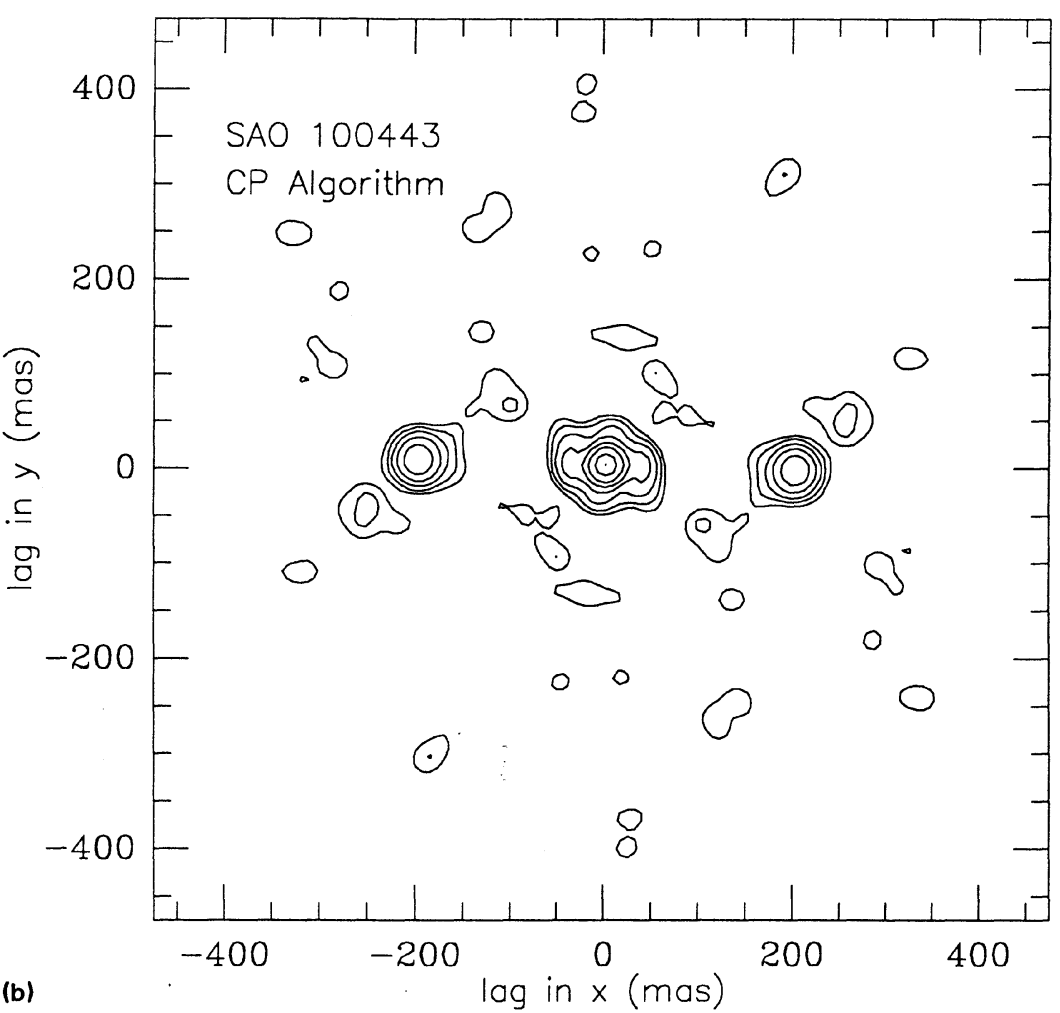

FIG. 6. (continued)

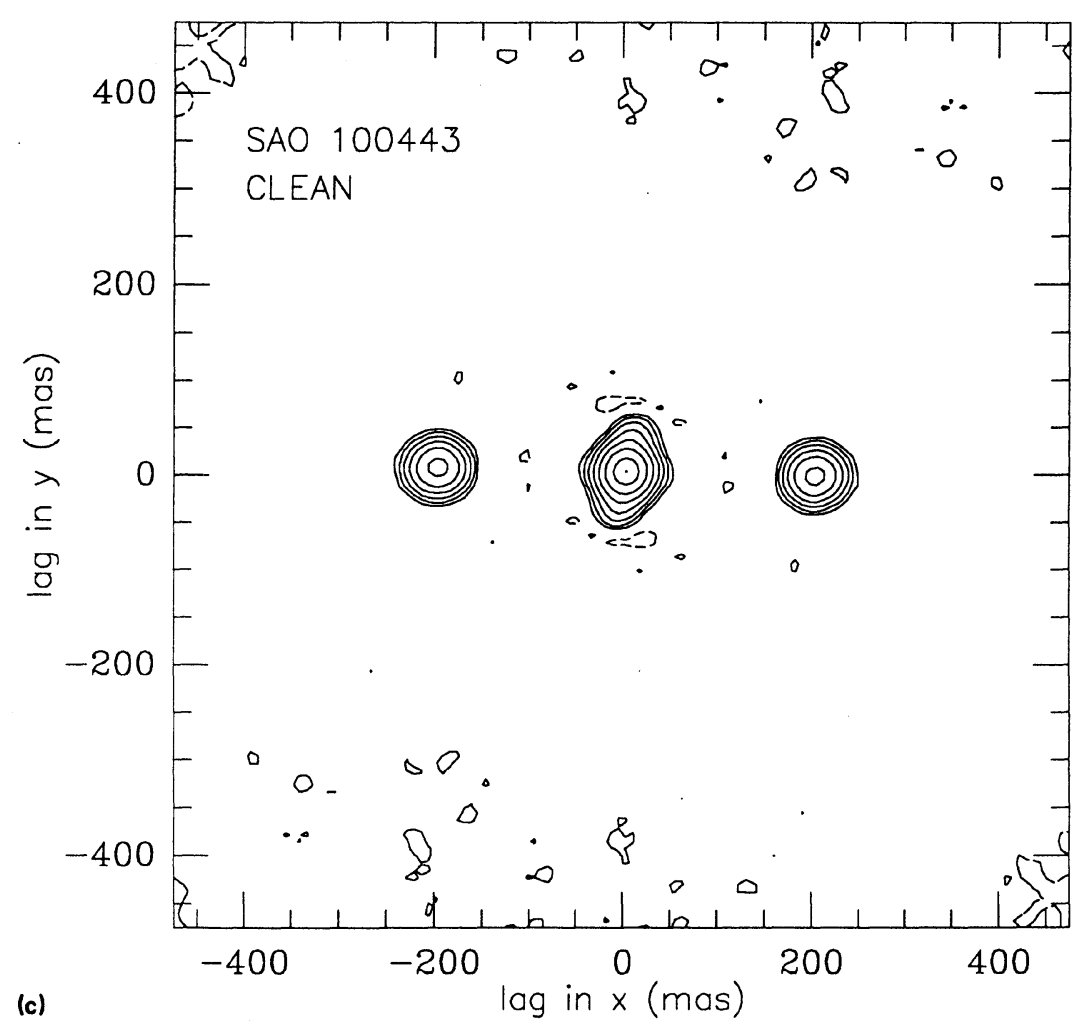

(C) American Astronomical Society - Provided by the NASA Astrophysics Data System 
spectral domain, although physically sensible, produce additional noise in both domains, and it is unclear how such noise behaves under the Fourier transform.

We have begun to evaluate the differences between the CLEAN and CP algorithms for speckle interferometry on a number of different types of data, including infrared speckle data which are not photon limited, and we find that CLEAN in all cases shows improved performance over the CP algorithm, except where there is large low spatial frequency systematic noise in the data with significant power $(\sim 10 \%$ or more) compared to the signal. In these cases CLEAN tends to wander away from the signal region by finding maxima along the saddles or humps in the raw ACF that are associated with the systematic noise. Surprisingly, the CP algorithm seems remarkably immune to such systematics and will recover visibilities for binary star systems where CLEAN utterly fails. Thus in cases where such noise (which is usually not due to a convolution process ) cannot be avoided or removed before the application of CLEAN, CP or a similar iterative algorithm may be the only hope for amplitude restoration.

We have found comparison of our results with other published results in speckle amplitude or ACF recovery to be virtually impossible. The reasons for this are that there are presently no widely used standards for quantifying speckle results, and where we have found quotations of recovered magnitude differences, which give an indication of the dynamic range of the recovered ACF, they are usually for telescopes significantly smaller than the $5 \mathrm{~m}$, or for data which are not photon limited. Also, the discussion above should indicate the dangers in using the recovered $\Delta m$ as a measure of the data quality.

Finally we note that for computation-bound applications CLEAN is significantly more efficient than algorithms which iterate between spectral and ACF domains. For example, the $C P$ algorithm requires two FFTs per iteration and $\sim 10$ iterations for convergence in the binary star recoveries, implying floating point operations of order $200 M \log _{2} M$ for an $M$-pixel image. For CLEAN in our data, at most $\sim 40$ peakfinding iterations were needed to recovery the binary star ACFs, each requiring $O(M)$ operations. For $M=128^{2}$ the entire process requires $\sim 1$ min on a SUN 3/50 workstation. A $128^{2}$ FFT on the same machine requires about $15 \mathrm{~s}$, implying a factor of order 5-10 in improved efficiency of CLEAN over the CP algorithm in this case.

\section{CONCLUSIONS}

We have successfully applied the CLEAN algorithm to the problem of deconvolving atmospheric seeing effects from the autocorrelations of speckle interferograms, recovering diffraction-limited autocorrelations of a number of binary star systems. CLEAN was also effective in recovering a high-resolution, though not diffraction-limited, autocorrelation of asteroid 2 Pallas. We find the algorithm to be straightforward to apply to speckle deconvolution, yielding high-quality results even under less than optimal seeing conditions, and capable of achieving dynamic ranges of as much as 70:1 at a resolution equivalent to 30 mas in the image domain.

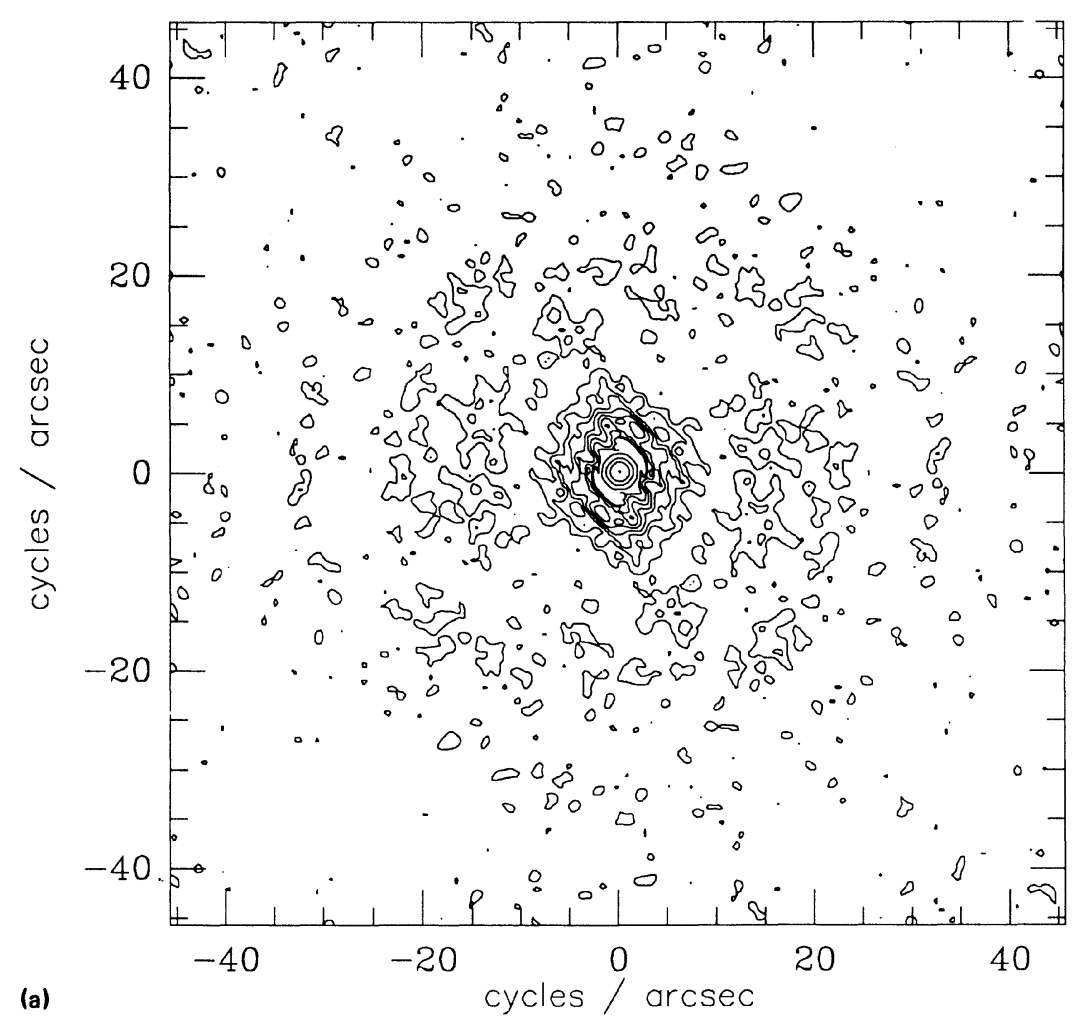

FIG. 7. (a) Similar to Fig. 6(a), for $\beta$ LMi. (b) Similar to Fig. 6(b); dynamic range is now about 10:1 with significant distortion of the central lobe. (c) Similar to Fig. 6(c), with a dynamic range of $30: 1$. The distortion of the central lobe seen in (b) is absent here. 
305 GORHAM ET AL. : SPECKLE INTERFEROGRAMS

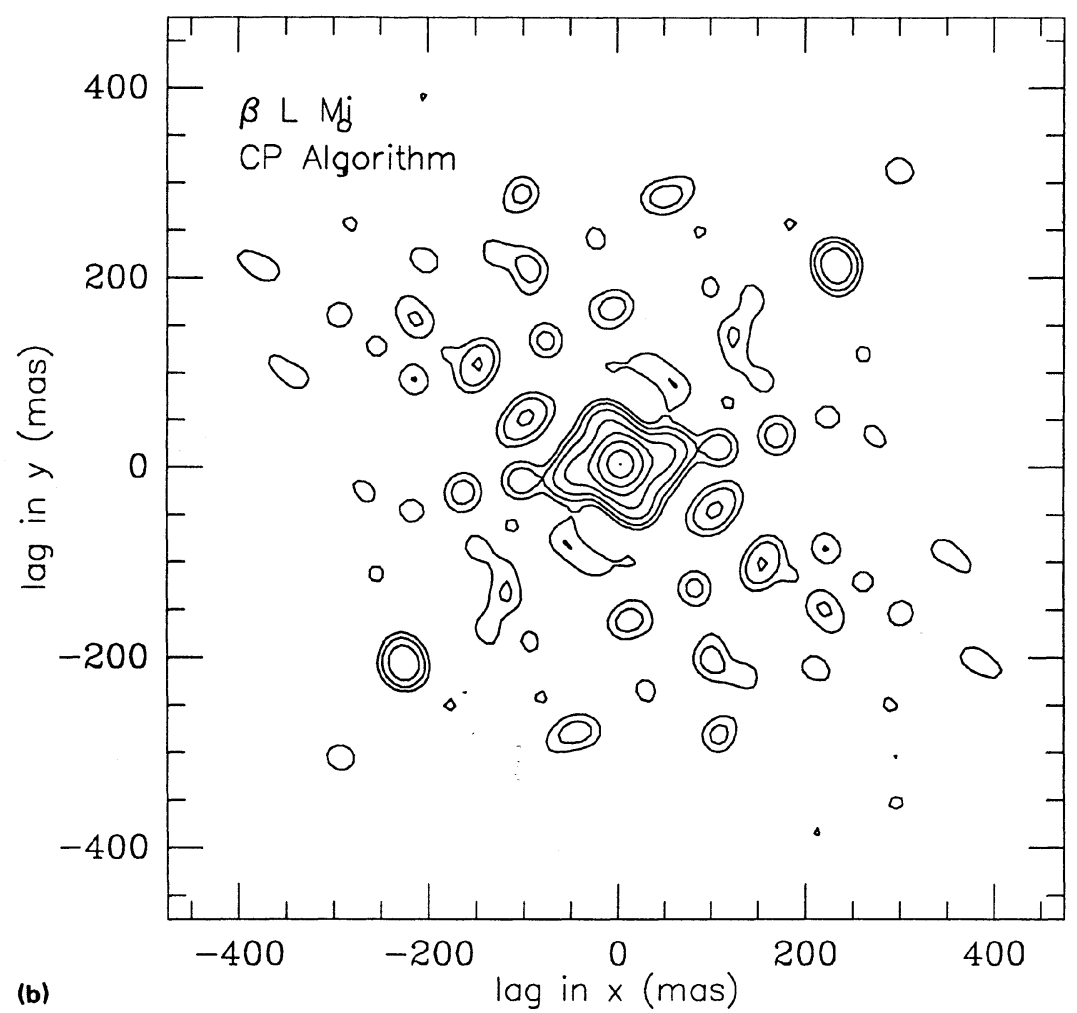

FIG. 7. (continued)

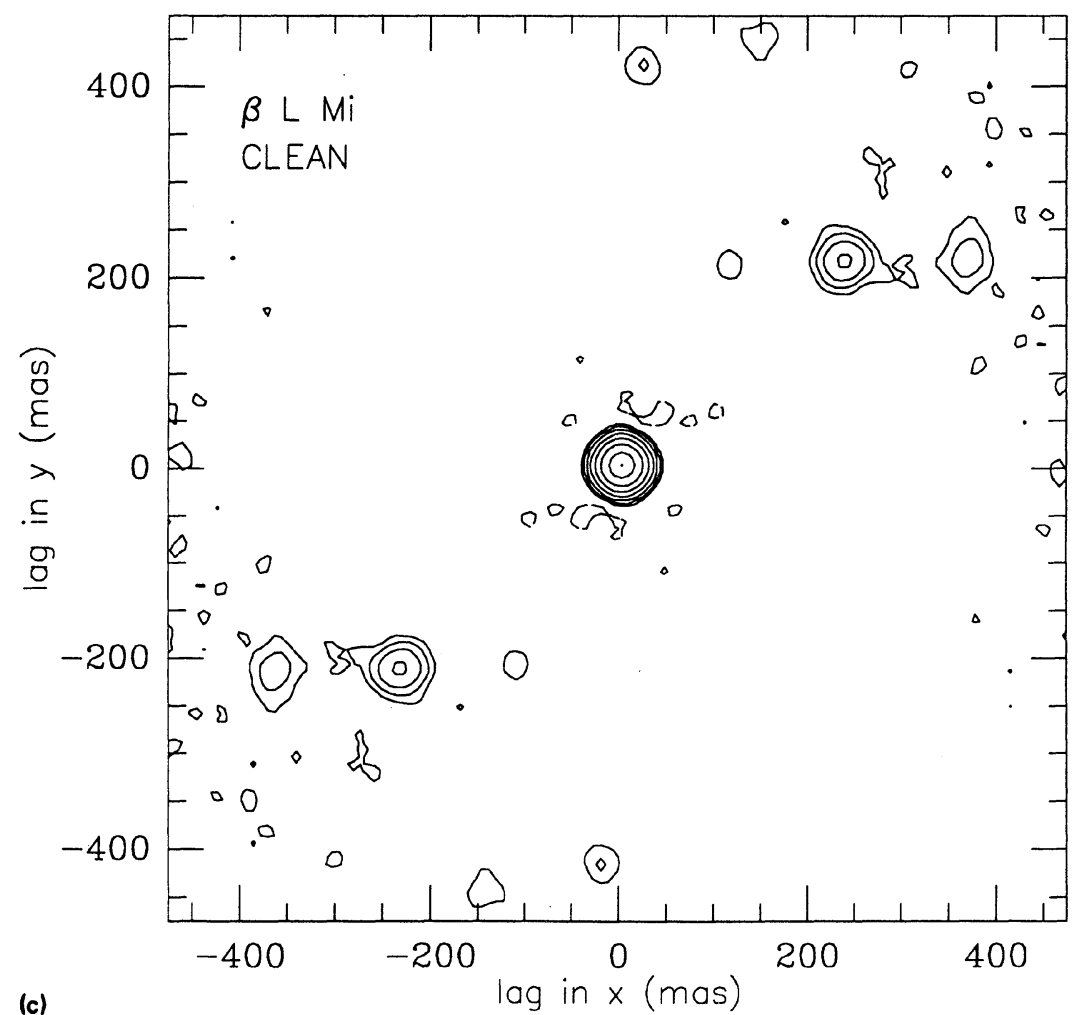

(C) American Astronomical Society - Provided by the NASA Astrophysics Data System 
We thank the W. M. Keck Foundation for their financial support, the Concurrent Computing Center at Caltech for providing the NCUBE computer time for data reduction, and S. Kulkarni, T. Nakajima, G. Neugebauer, and A. III Readhead for useful discussion and comments. We also thank R. Cook, G. Smith, A. Lee, and the staff of Palomar Observatory for their invaluable help with the hardware and observations. This work was supported in part by DOE Grant No. DE-FG03-85ER25009 and NSF Grant No. AST8351736.

\section{REFERENCES}

Clampin, M., Crocker, J., Paresce, F., and Rafal, M. (1988). Rev. Sci. Instrum. 59, 1269.

Clark, B. G. (1980). Astron. Astrophys. 89, 377.

Cornwell, T. J. (1983). Astron. Astrophys. 121, 281.

Cornwell, T. J. (1986). In Synthesis Imaging, edited by R. Perley, F. Schwab, and A. Bridle (NRAO, Green Bank), p. 109.

Dainty, J. C., and Greenaway, A. H. (1979). J. Opt. Soc. Am. 69, 786.

Drummond, J. D., and Hege, E. K. (1988). Icarus 73, 1.

Ebstein, S. (1987). Appl. Opt. 26, 1530.

Fox, G. C., Johnson, M. A., Lyzenga, G. A., Otto, S. W., Salmon, J. K., and Walker, D. W. (1988). Solving Problems on Concurrent Processors (Prentice-Hall, Englewood Cliffs).

Gorham, P. W., Ghez, A. M., Kulkarni, S. R., Nakajima, T., Neugebauer, G., Oke, J. B., and Prince, T. A. (1989). Astron. J. 98, 1783.

Greenaway, A. H., and Dainty, J. C. (1980). Opt. Commun. 35, 307.

Hogbom, J. A. (1974). Astron. Astrophys. Suppl. 15, 417.

Labeyrie, A. (1970). Astron. Astrophys. 6, 85.

Lebofsky, L. A., Sykes, M. V., Tedesco, E. F., Veeder, G. J., Matson, D. L., Brown, R. H., Gradie, J. C., Feierberg, M. A., and Rudy, R. J. (1986). Icarus 68, 239.

Matson, D. L., Veeder, G. J., Walker, R. G., Fowler, J. W., Chillemi, J. R.,
Lebofsky, L. A., and Tedesco, E. F. (1986). In IRAS Asteroid and Comet Catalog, edited by D. L. Matson (JPL, Pasadena).

McAlister, H. A., and Hartkopf, W. I. (1984). Catalog of Interferometric Measurements of Binary Stars (Georgia State University, Atlanta).

McAlister, H. A., and Hartkopf, W. I. (1988). Second Catalog of Interferometric Measurements of Binary Stars, Center for High Angular Resolution Astronomy (Georgia State University, Atlanta).

Nakajima, T., Kulkarni, S. R., Gorham, P. W., Ghez, A. M., Neugebaer, G., Oke, J. B., Prince, T. A., and Readhead, A. C. S. (1989). Astron. J. 97, 1510.

Nisenson, P. (1988). In Proceedings of the NATO Advanced Study Institute on Diffraction-Limited Imaging with Very Large Telescopes, edited by D. M. Alloin and J.-M. Mariotti (Kluwer, Dordrecht), p. 157.

Roddier, F. (1981). In Progress in Optics XIX, edited by E. Wolf (NorthHolland, Amsterdam), p. 281.

Schwarz, U. J. (1978). Astron. Astrophys. 65, 345.

Schwarz, U. J. (1979). In Image Formation from Coherence Fluctuations in Astronomy, edited by C. van Schooneveld (Reidel, Dordrecht), p. 261.

Steer, D. G., Dewdney, P. E., and Ito, M. R. (1984). Astron. Astrophys. $137,159$. 